\title{
A NOVEL TREATMENT FOR TEMPOROMANDIBULAR JOINT INTERNAL DERANGEMENT: A RANDOMIZED CONTROLLED CLINICAL STUDY
}

\author{
Mohammed Ahmed Elsholkamy*
}

\begin{abstract}
Background: Temporomandibular joint (TMJ) disorders are among the common causes of facial pain. Internal derangement is the most common intra-articular TMJ disorder.

Objective: The aim of this study was to evaluate the efficacy of ozone application in arthrocentesis of temporomandibular joints with internal derangement.

Patients and methods: Thirty patients were recruited in this study. They presented with limited function and pain related to the TMJ. They were randomly and equally divided in two groups; group 1 (study group), subjected to arthrocentesis using ozonized water and group 2 (control group), subjected to arthrocentesis with saline only. Visual analogue scale (VAS) pain scores and mouth opening were assessed preoperatively and at different intervals postoperatively.
\end{abstract}

Results: Pain levels decreased in both groups however, a significant decrease in the study group was noted after one month and one year postoperatively. Maximal mouth opening improved for all patients in both groups and the significant increase was reported in the study group after one month, six months, and one year postoperatively.

Conclusion: Ozone showed clinical efficacy during arthrocentesis of temporomandibular joint internal derangements.

KEYWORDS - Ozone; Temporomandibular; Arthrocentesis

\section{INTRODUCTION}

The temporomandibular joint is a synovial diarthroidal joint lined internally by synovial membrane that secretes synovial fluid. This fluid in turn acts along with the TMJ ligaments as cushion and shock absorber and lubricates the joint. It also supplies nutrients to the disc and non-vascularized structures within the joint. ${ }^{1,2}$

The joint is involved in a group of physiological, personal, and social communicational functions so that any limitation of function or related pain is so distressful to the patient. The rich sensory

* Assistant professor of Oral and Maxillofacial Surgery, Faculty of Dentistry, Suez Canal University. 
innervation of the head and neck as well as referred pain add to the complexity of the TMJ disorders. The TMJ disorders are among the common causes of facial pain where internal derangement is the most common intra-articular TMJ disorder. The internal derangement etiology is multifactorial which may be traumatic, parafunctional habits, or iatrogenic. Usually, the articular disc slips anteriorly and this displacement could be reducible or nonreducible. Furthermore, painful lock of the jaw might develop which if not resolved will lead to severe limitation of mouth opening and pain on the affected side. Failure to seek proper treatment or remove the most obvious cause can deteriorate the condition to arthritic degenerative disease and even disc perforation. ${ }^{3-5}$

Nitzan et al in the early nineties described arthrocentesis as a simple less invasive form of TMJ internal derangement manipulation. ${ }^{6}$ Their objective was to release the adhesions in the upper joint space between the articular fossa and the disc. This was better achieved via irrigating the superior joint space at first under hydraulic pressure using several maneuvers. ${ }^{6,7}$ The infusion and outflow of around $300 \mathrm{~mL}$ of irrigating solution through the upper joint space can help release the adhesions and wash out the inflammatory mediators and metabolites accumulated inside. ${ }^{6,8}$ However, the active jaw manipulation throughout the whole procedure is a key factor for the success to release the "stuck disc " to increase the limited mouth opening besides helping the outflow washing of pain stimulating inflammatory mediators. ${ }^{6,9}$

The medical applications of ozone are promising and offer different therapeutic benefits. ${ }^{10}$ Ozone consists of three oxygen atoms and exists as a naturally occurring compound. It has an antiinflammatory effect via the ability of oxidizing compounds having double bonds such as arachidonic acid and prostaglandins that play a major role in inflammatory response. Ozone also exerts an analgesic effect through oxidation of Algopeptides that determine the intensity of pain response by acting on free nerve endings after tissue damage. ${ }^{11}$ The current study aimed to assess the efficacy of ozone therapy in arthrocentesis of TMJ in cases of internal derangement.

\section{PATIENTS AND METHODS}

\section{Patients sampling and study design}

This study included thirty patients who were recruited from those presenting at the outpatient clinic of oral and maxillofacial surgery department, Faculty of dentistry, Suez Canal University and private practice. They were divided randomly and equally into two groups. Group 1 (study group), where fifteen patients underwent arthrocentesis using ozonized water. Group 2 (control group), where fifteen patients underwent arthrocentesis using saline solution.

All patients complained of pain related to the TMJ region, limitation of mouth opening, and jaw dysfunction. The main inclusion criterion followed ResearchDiagnostic CriteriaforTemporomandibular Disorders (RDC/TMD). ${ }^{12}$ The cases included were those having internal derangement of the TMJ and disc displacement without reduction.

Exclusion criteria included patients with TMJ arthritis, condylar hyperplasia or hypoplasia, and previous TMJ arthroscopy or lavage. Patients with pain and limited mouth opening due to muscular origin only were also excluded from the present study.

\section{Preoperative assessment}

All patients signed an informed consent regarding their enrollment in the study. They were informed about the aim of the study, follow-up duration, and societal benefits. Pain intensity was assessed via the visual analogue scale (VAS) pain scores on a scale from 0 to 10 , where 0 represented 
no pain and 10 denoted the worst pain ever felt. Maximal unassisted mouth opening was measured interincisally (between the incisal edges of upper and lower incisors at the midline) using millimeter ruler. All patients had filled a printed questionnaire gathering information about the history of the chief complaint and the different signs and symptoms. All patients underwent magnetic resonance imaging (MRI) preoperatively (Figs. 1 and 2)

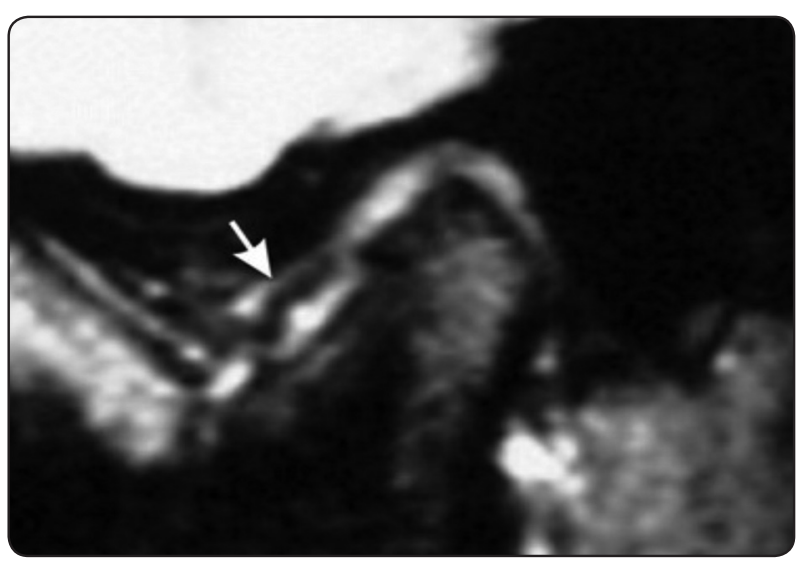

Fig. (1) A photograph of closed mouth MRI showing anteriorly displaced disc (white arrow)

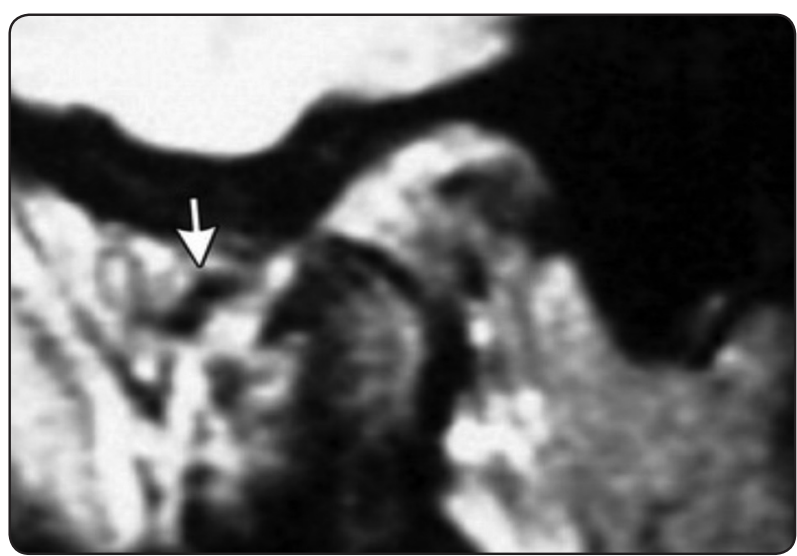

Fig. (2) A photograph of open mouth MRI showing anteriorly displaced disc without reduction (white arrow)

\section{Operative procedures:}

All patients signed an informed consent declaring their approval of undergoing the surgical procedures. The consent explained the surgical procedures, their potential complications, and alternative treatment. All cases were managed under local anesthesia via auriculotemporal nerve block using Articaine hydrochloride 4\% with 1:100.000 epinephrine (Ubistesin forte, 3M ESPE Germany). The patients were sitting in the supine position and the surgical field was scrubbed and disinfected using Providone-Iodine 10\% (Betadine, the Nile Co. Pharma, Cairo, Egypt licensed from Mundipharma AG, Basel, Switzerland). The same operator under aseptic conditions and following standardized protocols performed the arthrocentesis for the cases of both groups. Determination of the entry point of the first needle to the superior joint space was started first by drawing the canthus-tragus line and followed Talaat et al. guidelines. ${ }^{13}$ In cases where the canthus-tragus line was $70 \mathrm{~mm}$ or more, the first entry point was $10 \mathrm{~mm}$ in front of the tragus and $2 \mathrm{~mm}$ below the canthus-tragus line. On the other hand, when the canthus-tragus line was less than $70 \mathrm{~mm}$, the entry point was marked $7 \mathrm{~mm}$ in front of the tragus and $2 \mathrm{~m}$ below the canthus-tragus line. The second needle insertion point was $2 \mathrm{~mm}$ anterior to the former one and was for the outflow needle (Fig. 3).

In the control group, a 20-gauge needle was inserted at the first entry point then injection of $2 \mathrm{ml}$ saline solution was performed to increase the hydraulic pressure within the joint space. Jaw

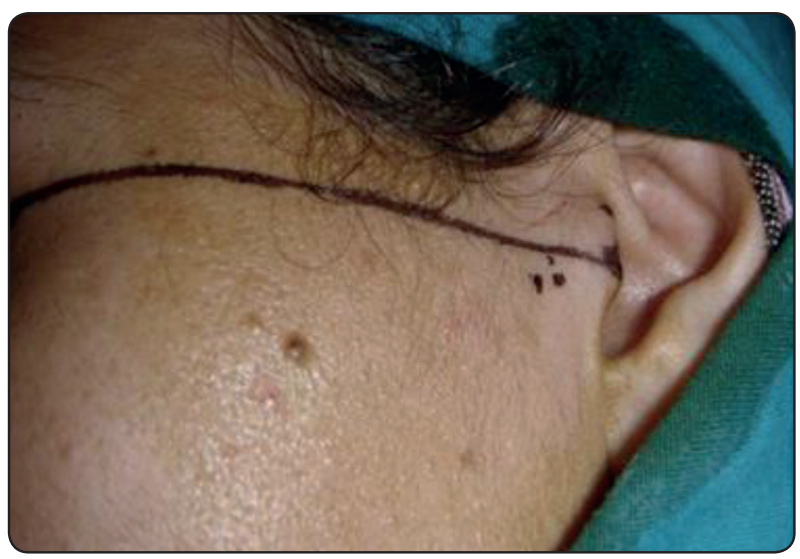

Fig. (3) A clinical photograph of the canthus-tragus line with the two points marked below. 
manipulation was performed by telling the patients to open and close to free the joint movement and the operator assisted the patients with this manipulation to get the maximum free movement. The second needle was inserted in the second point for the outflow of the solution. The joint was then washed with about $300 \mathrm{~mL}$ of saline solution injected into the upper joint compartment with instructing the patients to keep up the jaw movements. The needles were withdrawn and the patients were instructed to keep jaw opening and closing for a little while.

\section{Ozonized water preparation:}

In the study group, the operator followed the same procedures but ozonized water was employed in the lavage process instead of saline. Ozonized water was prepared by bubbling ozone in injectable distilled water (distilled water prepared for injection, EIPICO, Egypt). A standard glass bottle with $200 \mathrm{~mL}$ capacity was filled with the injectable distilled water then the water was bubbled with 70$\mu \mathrm{g} / \mathrm{mL}$ ozone for 30 minutes at room temperature not exceeding $25^{\circ} \mathrm{c}$. The medical ozone/oxygen gas mixture was produced by ozone generator (Humazon ${ }^{\circledR}$ Promedic, Humares GmbH, Bruchsal, Germany). The generator was supplied with medical pure oxygen via a medical oxygen tank where the generator was pre-adjusted to produce the previously mentioned ozone concentration (Fig. 4).

\section{Postoperative assessment:}

The clinical parameters assessed preoperatively, VAS pain scores and maximal unassisted mouth opening, were assessed at different sessions postoperatively. They were checked immediately after the procedure and after 2 days postoperatively. The patients were then recalled after one month, 6 months, and one year for further assessment of the same clinical parameters. The same clinician did the assessment for all cases and at different sessions and the data collected was saved, tabulated, and statistically tested.

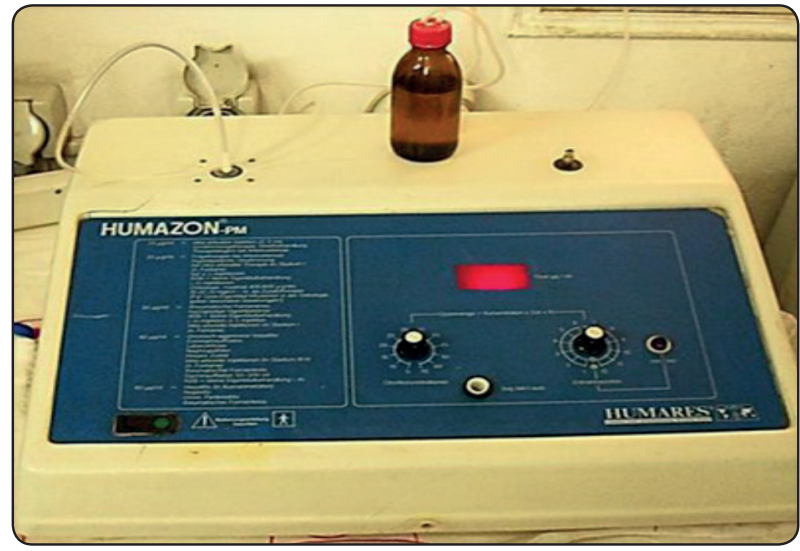

Fig. 4. Ozone generator ((Humazon® Promedic, Humares $\mathrm{GmbH}$, Bruchsal, Germany) and the fed glass bottle connected via inlet and outlet tubes.

\section{RESULTS}

The present study included thirty patients, twenty one females and nine males aged between 19 and 43 years. In group one, the patients ranged in age from 19-37 years (mean $24.923 \pm 6.256$ ). In group two, the patients ranged in age from 20-43 years (mean $26.357 \pm 5.321$ ). Group one included 11 females $(73 \%)$ and 4 males (27\%). Group two included 10 females (66.7 \%) and 5 males (33.4\%). There was a statistically significant majority in the number of female patients in both groups $(\mathrm{P}<0.005)$ while no statistical significance in sex predilection between both groups was noted.

There was a significant decrease in pain levels in both groups on comparing preoperative and all postoperative scores throughout the study. On comparing the postoperative pain levels between both groups, the study group showed more decrease rather than the control group. However, the statistical analysis revealed a significant decrease at the immediate postoperative assessment. The decrease after 2 days and 6 months was not statistically significant whereas, after 1 month and 1 year it was statistically significant $(P<0.05)$ (Table 1, Fig. 5).

The maximal mouth opening showed a statistically significant improvement in both 
groups when comparing preoperative and all postoperative measurements throughout the study. At immediately postoperative, the maximal mouth opening improved in both groups without statistical significant difference between them $(P=0.258)$. After two days, there was no statistical significant difference between both groups. The records of maximal mouth opening in both groups After 1 month, sixth months, and one year indicated a significant increase in the mouth opening in the study group in comparison with the control group with $\mathrm{P}$-value $(P<0.05),(P<0.03)$, and $(P<0.001)$ respectively (Table2, Fig 6).

TABLE (1) Analysis of the VAS pain scores for both groups throughout different assessment sessions of the study.

\begin{tabular}{|c|c|c|c|c|}
\hline Group & Assessment timing & Mean & SD & P-value \\
\hline \multirow[b]{3}{*}{ Group 1} & Pre-Operative & 6.133 & 1.069 & \\
\hline & Immediate Post-Operative & 0.943 & 0.714 & $<0.001 *$ \\
\hline & 2 Days postoperatively & 2.853 & 0.640 & $<0.001 *$ \\
\hline \multirow{3}{*}{ Study group } & 1 Month postoperatively & 0.000 & 0.000 & $<0.001 *$ \\
\hline & 6 Months postoperatively & 0.256 & 0.640 & $<0.001 *$ \\
\hline & 1 Year postoperatively & 0.518 & 0.637 & $<0.001 *$ \\
\hline \multirow{3}{*}{ Group 2} & Pre-Operative & 7.213 & 0.859 & \\
\hline & Immediate Post-Operative & 1.712 & 0.613 & $<0.001 *$ \\
\hline & 2 Days postoperatively & 2.561 & 0.659 & $<0.001 *$ \\
\hline \multirow{3}{*}{ Control group } & 1 Month postoperatively & 0.335 & 0.338 & $<0.001 *$ \\
\hline & 6 Months postoperatively & 0.456 & 0.638 & $<0.001 *$ \\
\hline & 1 Year postoperatively & 1.618 & 0.697 & $<0.001 *$ \\
\hline
\end{tabular}

Table (2) Analysis of the maximal mouth opening records for both groups throughout different assessment sessions of the study.

\begin{tabular}{|c|c|c|c|c|}
\hline Group & Assessment timing & Mean & SD & P-value \\
\hline \multirow[b]{3}{*}{ Group 1} & Pre-Operative & 28.713 & 3.189 & \\
\hline & Immediate Post-Operative & 38.911 & 4.123 & $<0.001 *$ \\
\hline & 2 Days postoperatively & 34.247 & 5.023 & $<0.001^{*}$ \\
\hline \multirow{3}{*}{ Study group } & 1 Month postoperatively & 40.933 & 3.345 & $<0.001 *$ \\
\hline & 6 Months postoperatively & 41.512 & 3.652 & $<0.001 *$ \\
\hline & 1 Year postoperatively & 41.512 & 3.652 & $<0.001 *$ \\
\hline \multirow{3}{*}{ Group 2} & Pre-Operative & 29.134 & 4.243 & \\
\hline & Immediate Post-Operative & 40.689 & 2.537 & $<0.001 *$ \\
\hline & 2 Days postoperatively & 36.233 & 3.120 & $<0.001 *$ \\
\hline \multirow{3}{*}{ Control group } & 1 Month postoperatively & 38.834 & 2.217 & $<0.001 *$ \\
\hline & 6 Months postoperatively & 38.917 & 2.357 & $<0.001 *$ \\
\hline & 1 Year postoperatively & 37.700 & 3.627 & $<0.001 *$ \\
\hline
\end{tabular}




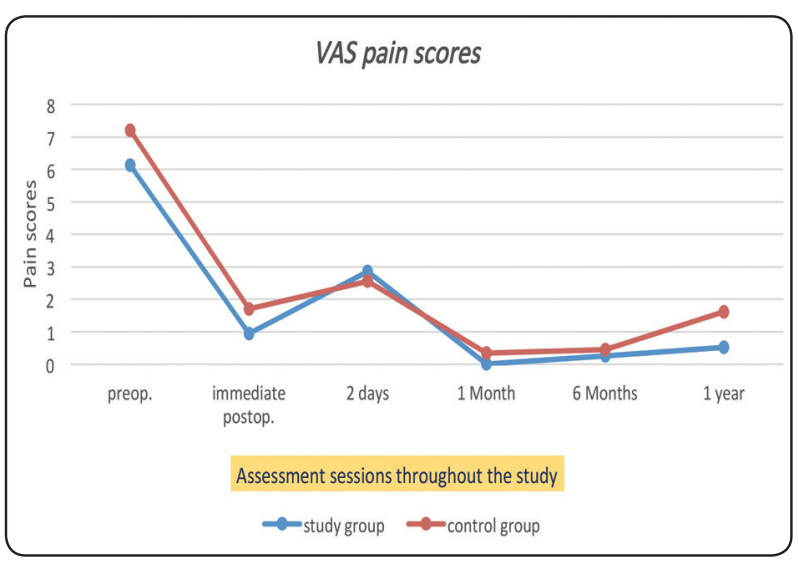

Fig. (5) Graph showing the change in pain levels for both groups throughout the study assessment sessions.

\section{DISCUSSION}

Thirty patents with disc displacement without reduction underwent arthrocentesis in this study where half of them had their joints washed with ozonized water and the other half with saline. The postoperative assessment revealed statistically significant decrease in the VAS pain scores in both groups when compared to the preoperative values. This decrease in pain levels in most cases was attributed to the efficacy of the arthrocentesis procedure and the active jaw manipulation via opening and closing. This lavage in turn had a positive impact on washing away and diluting the inflammatory mediators as well as releasing the adhesions between the mandibular fossa and the disc. ${ }^{14,15}$

However, the study group using the ozonized water showed significant decrease in pain levels particularly after 1 month and 1 year. This reflected the ozone effect and its relatively long lasting pain relieving action. Ozone possesses some clinically relevant actions such as anti-inflammatory, antimicrobial, analgesic, immuno-modulatory and stimulatory actions. ${ }^{10,11}$ The author believes that one of the most important effects of ozone is the antihypoxic. It can help tissue hyper oxygenation with neoangiogenesis, which accelerates healing, and

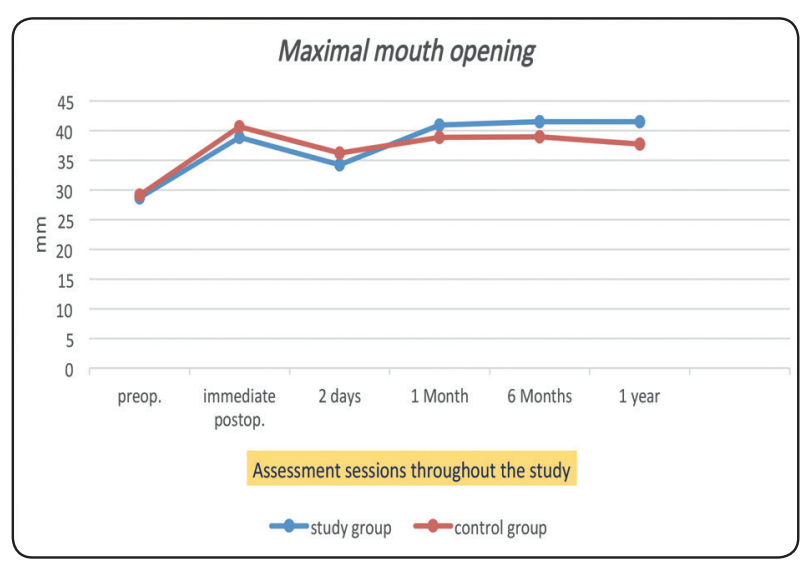

Fig. (6) Graph showing the change in maximal mouth opening for both groups throughout the study assessment sessions.

repair in general. ${ }^{16}$ This might be responsible for the relatively long-term improvement of the study group cases.

All patients in both groups had gained highly significant improvement in the unassisted maximal mouth opening from the immediate postoperative follow up to one year. This also validates the efficacy of arthrocentesis to treat cases of disc displacement without reduction via disc mobilization and release of adhesions. ${ }^{14,15}$ Moreover, the decrease in pain levels helped the patients to open confidently and freely without having the guarding action of fear of having pain on opening as they used to experience. The comparison between both groups regarding the mouth opening did not show any statistical difference at first two postoperative sessions. However, the study group showed statistically significant improvement in the mouth opening after one month, six months, and one year. The ozone had its positive impact on such findings and this was in accordance with several publications and reviews. ${ }^{10,11,16,17,18}$ The assessment parameters used for follow up of the cases in the current study were believed to be sufficient by the author. There might be some debate concerning the subjectivity and sensitivity of the VAS scores being affected by the culture, ethnicity, age, and psychological status. 
However, most studies and publications refer to VAS pain scores, jaw function, and mouth opening as the convenient and feasible clinical parameters for assessment of arthrocentesis outcome. ${ }^{14,15}$

Benvenuti described his own experience in 2006 concerning management of acute and chronic diseases of large joints (shoulder, knee, and hip) by intra articular and periarticular injections of micro doses $(15 \mu \mathrm{g} / \mathrm{ml}-25 \mu \mathrm{g} / \mathrm{ml})$ of oxygen ozone mixture. He reported that the patients experienced resolution of joint pain besides good functional recovery of their daily activities. ${ }^{17}$ Mishra et al. conducted a comparative study on the effect of intra-articular ozone injection versus corticosteroids (methylprednisolone) in knee osteoarthritis. They achieved $80 \%$ success rate at three and six months in the ozone group whereas, the corticosteroid group achieved a lesser success rate $(60 \%)$. They concluded that ozone intra-articular injection relieved pain, disability, and stiffness better than corticosteroids. ${ }^{18}$ This can be explained by the hyper-oxygenation and reparative capacity of ozone. ${ }^{16}$ The results of the current study agreed with these findings and even yielded better success rate after longer follow up.

Several studies have utilized different forms of ozone therapy in treatment of TMJ disorders. A high frequency, bio-oxidative therapy via omega probes were applied to the affected joints. ${ }^{19}$ Those probes emit energy around the treated area, which splits diatomic oxygen in the region into singular atomic oxygen and ozone. This action reaches and affects subcutaneous, deep tissues yet it needs to be repeated for several sessions per week for a considerable amount of time. This form of ozone therapy proved to be effective than ketoprofen medication for relieving TMJ related pain. ${ }^{19}$ The same principle was applied to the trigger points in masticatory muscles for managing cases of myofascial pain and it proved effectiveness in relieving the symptoms..$^{20}$ Ozone gas also had been tried as an intraarticular injection for internal derangement cases and the outcome was satisfactory and promising. ${ }^{21}$ The current study shared the clinical improvement and the promising outcome with the previously mentioned studies yet the methodology and rationale were entirely different.

The present study made use of the concept postulated by Bocci that ozone could dissolve physically in pure water if optimum temperature, pressure, and ozone concentration exist. In this situation, ozone will not react and when kept in tight glass bottle it could remain active and potent for two days. ${ }^{11}$ This necessitated preparing the ozonized water after scheduling the arthrocentesis to be ready and efficient. The use of saline as a solvent for ozone was not applicable as it would have produced toxic hypochlorite because ozone would react immediately when dissolved in physiological saline. ${ }^{11}$ The presence of a vehicle that facilitated transforming ozone to an injectable solution led to its use as lavage solution for TMJ internal derangement arthrocentesis. Ozone application necessitates equipment and generator, the shelf life of the solution is short, besides appropriate handling and storage should be implemented to guarantee active solution for satisfactory outcome. The author of the current study believed that combining the ozone therapy application with arthrocentesis had provided the benefits of both for a better outcome of the treatment. From the current data, it has been concluded that arthrocentesis is an effective less invasive procedure for treatment of TMJ internal derangement and the ozone therapy proved clinical efficacy when used in combination. Moreover, ozonized water is a clinically convenient applicable form of ozone therapy in the superior joint space for managing TMJ internal derangement.

\section{DISCLOSURE}

The author declares explicitly that there is no conflict of interest regarding the present study. 


\section{REFERENCES}

1. Bouloux GF. Temporomandibular joint pain and synovial fluid analysis: A review of the literature. J Oral Maxillofac Surg. 2009; 67 (11): 2497-504

2. Poveda-Roda R, Díaz-Fernández JM, Hernández-Bazán S, JiménezSoriano Y, Margaix M, Sarrión G. A review of temporomandibular joint disease (TMJD). Part II: Clinical and radiological semiology. Morbidity processes. Med Oral Patol Oral Cir Bucal. 2008 Feb1; 13(2):E102-9.

3. Okeson JP. Causes of functional disturbances in the masticatory system. In Okeson JP, editor: Management of Temporomandibular Disorders and Occlusion, 7th Edition, 2012, Mosby.

4. Badel T, Marotti M, Kern J, Laskarin M. A quantitative analysis of splint therapy of displaced temporomandibular joint disc. Ann Anat. 2009; 191(3): 280-7.

5. Manfredini D, Guarda-Nardini L, Winocur E, Piccotti F, Ahlberg J, Lobbezoo F. Research diagnostic criteria for temporomandibular disorders: A systematic review of axis I epidemiologic findings. Oral Surg Oral Med Oral Pathol Oral Radiol Endod. 2011; 112(4):453-62.

6. Nitzan DW, Dolwick MF, Martinez GA. Temporomandibular joint arthrocentesis: A simplified treatment for severe, limited mouth opening. J Oral Maxillofac Surg. 1991; 49: 1163-67.

7. Alkan A and Kilic E. A new approach to arthrocentesis of the temporomandibular joint. Int J Oral Maxillofac Surg. 2008; 38: 85-86.

8. Alpaslan G.H, Alpaslan C. Efficacy of temporomandibular joint arthrocentesis with and without injection of sodium hyaluronate in treatment of internal derangements. J Oral Maxillofac Surg. 2001; 59: 613-18.

9. Laskin DM. Arthrocentesis for the Treatment of Internal Derangements of the Temporomandibular Joint. Alpha Omegan. 2009; 102, 46-50.

10. Bocci VA. Biological and clinical effect of ozone: Has ozone therapy any future in medicine? Br J Biomed Sci. $1999 ; 56: 270-279$.

11. Bocci VA. Scientific and medical aspects of ozone therapy.
State of the art. Arch Med Res. 2006; 37(4):425-35

12. Maydana AV, Tesch RS, Denardin OVP, Ursi WJS, Dworkin SF. Possible etiological factors in temporomandibular disorders of articular origin with implications for diagnosis and treatment. Dental Press J Orthod. 2010; 15(3): 78-86.

13. Talaat WM, McGraw TA, and Klitzman B. Relationship between the canthal-tragus distance and the puncture point in temporomandibular joint arthroscopy. Int J Oral Maxfac Surg. 2010; 39: 57-60.

14. Cavalcanti do EgitoVasconcelos B, Bessa-Nogueira RV, Rocha NS. Temporomandibular joint arthrocententesis: Evaluation of results and review of the literature. Braz $\mathrm{J}$ Otorhinolaryngol. 2006; 72(5):634-8.

15. Monje-Gil F, Nitzan D, González-Garcia R. Temporomandibular joint arthrocentesis. Review of the literature. Med Oral Patol Oral Cir Bucal. 2012; 1:17(4): 575-81.

16. Sushma Das Application of Ozone Therapy in Dentistry. Indian journal of dental advancements IJDA. 2011; 3(2): 538-42.

17. Benvenuti P. Oxygen-Ozone Treatment of the Knee, Shoulder and Hip: A Personal Experience. RivistaItaliana di Ossigeno-Ozonoterapia. 2006; 5: 135-144.

18. Mishra SK, Pramanik R, Das P, Das PP, Palit AK, Roy J, Haldar RN. Role of intra-articular ozone in osteo-artheritis of knee for functional and symptomatic treatment. Indian journal of physical medicine and rehabilitation. IJPMR. 2011; 22: 65-69.

19. Doğan M, Özdemir Doğan D, Düger C. Özdemir Kol İ, Akpınar A, Mutaf B, Akar T. Effects of High-Frequency Bio-Oxidative Ozone Therapy in Temporomandibular Disorder-Related Pain. Med Princ Pract. 2014; 23: 507-510

20. Celakil T, Muric A, Gokcen Roehlig B, Evlioglu G, Keskin H. Effect of high-frequency bio-oxidative ozone therapy for masticatory muscle pain: A double-blind randomized clinical trial. J Oral Rehabil. 2017 Jun; 44(6): 442-451.

21. Daif ET. Role of intra-articular ozone gas injection in the management of internal derangement of the temporomandibular joint. Oral Surg Oral Med Oral Pathol Oral Radiol. June 2012; 113(6): e10-e14 